\title{
Sleep Modulation of Neuroendocrine Function: Developmental Changes in Gonadotropin-Releasing Hormone Secretion during Sexual Maturation ${ }^{1}$
}

\author{
HAL LANDY, PAUL A. BOEPPLE, M. JOAN MANSFIELD, PEGGY CHARPIE, \\ DAVID I. SCHOENFELD, KATHLEEN LINK, GLORIA ROMERO, JOHN D. CRAWFORD, \\ JOHN F. CRIGLER, JR., ROBERT M. BLIZZARD, AND WILLIAM F. CROWLEY, JR. \\ Reproductive Endocrine Unit and the Vincent Memorial Research Laboratories; Departments of Medicine, \\ Gynecology, and the Pediatric Endocrinology Unit of the Children's Service, Massachusetts General Hospital, \\ Boston, Massachusetts 02114; Divisions of Endocrinology and Adolescent and Young Adult Medicine, \\ Department of Medicine, Children's Hospital Medical Center, Boston, Massachusetts 02115; and Department of \\ Pediatrics, University of Virginia Medical Center, Charlottesville, Virginia 22908
}

\begin{abstract}
To assess sleep-associated changes in gonadotropin-releasing hormone secretion during sexual maturation, we studied nighttime and daytime patterns of $\mathrm{LH}$ and FSH secretion in two groups with qualitatively similar sex steroid levels: girls with central precocious puberty and young adult women in the early follicular phase of an ovulatory menstrual cycle. In the girls with central precocious puberty, all indices of $\mathrm{LH}$ secretion were significantly higher at night than during the day (mean LH levels, $12 \pm$ 2 versus $5 \pm 1 \mathrm{IU} / \mathrm{L}, p \leq 0.01$; LH pulse amplitude $16 \pm$ 2 versus $7 \pm 1 \mathrm{IU} / \mathrm{L}, p \leq 0.01$; and $\mathrm{LH}$ pulse frequency $0.70 \pm 0.05$ versus $0.35 \pm 0.08$ pulse/patient $-h, p \leq 0.01$ ). Girls with a history of menses, who were presumably the most mature, lacked this diurnal variability. Mean nocturnal FSH levels were only slightly higher than daytime levels $(7.6 \pm 0.5$ versus $7.2 \pm 0.5 \mathrm{IU} / \mathrm{L}, p \leq 0.05)$ resulting in alternating periods of $\mathrm{LH}$ (nighttime) and FSH (daytime) predominance in this pubertal population. In contrast, the adult women had lower mean gonadotropin levels and $L H$ pulse frequencies at night than during the day (mean LH $7 \pm 1$ versus $10 \pm 1 \mathrm{IU} / \mathrm{L}, p \leq 0.05$; mean FSH $9 \pm 1$ versus $10 \pm 1 \mathrm{IU} / \mathrm{L}, p \leq 0.05 ; \mathrm{LH}$ pulse frequency $0.40 \pm 0.08$ versus $0.70 \pm 0.10$ pulse/patient-h, $p \leq 0.05$ ) and often (six of eight) demonstrated striking suspension of gonadotropin-releasing hormone secretion during sleep. The smaller changes in FSH again resulted in periods of relative LH (daytime) and FSH (nighttime) predominance. When between-group comparisons were made, the girls with central precocious puberty differed significantly from the women in the early follicular phase with respect to each index of gonadotropin secretion except for daytime LH pulse amplitude. Thus, neuroendocrine maturation in the human female appears to be characterized by changes in both the pattern of gonadotropin-releasing hormone secretion and the daily alternating periods of relative $\mathbf{L H}$ and FSH predominance in response to sleep. (Pediatr Res 28: 213-217, 1990)
\end{abstract}

Received January 16, 1990; accepted May 1, 1990.

Correspondence and reprint requests: Dr. Hal Landy, Reproductive Endocrine Unit, Massachusetts General Hospital, Boston, MA 02114.

Supported in part by NIH Grants HD 18169, HD 07277, RR 01066, RR 02172 , and RR 08847.

Presented in part at the American Pediatric Society and the Society for Pediatric Research meetings, Washington, DC, May 1988.

\section{Abbreviations}

GnRH, gonadotropin-releasing hormone

CPP, central precocious puberty

EFP, early follicular phase

DSIP, delta sleep-inducing peptide

Sleep is an important neuroendocrine modulator of hypothalamic function. With the onset of puberty, a marked increase in sleep-entrained, GnRH-induced gonadotropin pulses occurs (1). After complete maturation of the reproductive axis, cyclic changes in hypothalamic-pituitary-gonadal axis function result in serum estradiol levels during the EFP of the menstrual cycle that approximate those of girls during puberty. Despite this comparable gonadal steroid milieu, women in the EFP exhibit a strikingly different response to sleep. In contrast to pubertal girls, women in the EFP demonstrate sleep-associated slowing in the frequency of $\mathrm{GnRH}$-induced gonadotropin secretory events (2, 3 ). To further explore the changing impact of sleep upon neurosecretory dynamics across development, we examined the nighttime and daytime patterns of gonadotropin secretion in 39 girls with idiopathic CPP and contrasted them with those of eight young adult women studied during the EFP of an ovulatory cycle.

\section{MATERIALS AND METHODS}

Patients. Thirty-nine girls with idiopathic CPP were studied as described below. Patients with neurogenic precocity (i.e. centrally mediated precocious puberty with radiographic evidence of a CNS lesion) were excluded because of the potential impact of their CNS defects on neuroendocrine function. Four girls with CPP who otherwise met inclusion criteria but whose progesterone levels were $>3.0 \mathrm{nmol} / \mathrm{L}$ and therefore suggestive of the presence of a functioning corpus luteum were also excluded from the analysis because of the known effect of progesterone on slowing the frequency of GnRH secretion (4). Previously published data from eight normal women in the EFP of an ovulatory menstrual cycle were reanalyzed for comparison with the girls with CPP (3). This phase of the menstrual cycle was chosen because estradiol and progesterone levels are at their lowest levels and the sex steroid milieu thus is most comparable to that seen in the pubertal group. 
Protocol. All studies were approved by a committee on human experimentation at each institution.

Girls with CPP. Informed parental consent was obtained for each girl with CPP before enrollment in the study. Patients were admitted to the Clinical Research Centers of the Massachusetts General Hospital, Children's Hospital, Boston or the University of Virginia between June 1981 and July 1986. Breast development and pubic hair were scored according to the method of Tanner and Marshall (5) and vaginal smears for calculation of a maturation-index score (6) were obtained. Blood samples for gonadotropins were drawn at 10 -min intervals from 2200 to $0200 \mathrm{~h}$ and from 1000 to $1400 \mathrm{~h}$ to assess gonadotropin pulsations. Sleep/wake status was recorded with each blood withdrawal and a serum estradiol level was obtained during the nighttime monitoring period. Cranial $\mathrm{CT}$ and $\mathrm{x}$-rays of the left hand and wrist for bone age (7) were also performed.

Women in EFP. Forty-eight normally cycling women had daily blood samples drawn for serum LH, FSH, estradiol, and progesterone during a complete menstrual cycle (3). On a predetermined day of the same cycle, each woman was admitted to the Clinical Research Center of the Massachusetts General Hospital where blood samples for gonadotropins were drawn at 10min intervals for 20-24 h and the sleep/wake status was recorded by an experienced observer with each blood withdrawal. In addition, blood samples for estradiol and progesterone were drawn every $6 \mathrm{~h}$ during the study from which a patient mean was determined. Menstrual cycle stage at the time of study was subsequently assigned in relation to the midcycle LH surge with the EFP of the menstrual cycle defined as the period from 9 to $13 \mathrm{~d}$ before the midcycle LH surge. In this manner, a cohort of eight women in the EFP was retrospectively identified.

Methods. Acknowledging that precise estimates of gonadotropin and thus GnRH pulse frequency are influenced by sampling frequency, RIA characteristics, and pulse identification criteria, our methods sought to optimize each of these variables. Because the number of identifiable pulses increases with increasingly frequent sampling, we chose a sampling frequency $(10 \mathrm{~min})$ designed to produce an acceptably low false positive pulse identification rate (8). Thus, we opted for short windows of intensive sampling rather than longer periods of less intensive sampling in the girls with CPP. The larger blood volumes in women in the EFP enabled us to sample both intensively and continuously for 24 h. Gonadotropin and sex steroid determinations were performed as previously described $(9,10)$. The LH assay uses a highly $\beta$-subunit-specific polyclonal antiserum with $2 \%$ crossreactivity with both intact FSH and the free $\alpha$-subunit of the gonadotropins. The FSH assay uses a highly conformationally specific polyclonal antiserum with less than $0.03 \%$ cross-reactivity with either intact $\mathrm{LH}$ or free $\alpha$-subunit. The mean intraassay coefficients of variation for $\mathrm{LH}$ and FSH were 7.4 and $8.1 \%$ and 7.2 and $8.7 \%$ for the girls with CPP and the women in the EFP, respectively. Gonadotropin pulses were defined as a $20 \%$ or greater increase from nadir to peak, corresponding to approximately 3 times the intraassay coefficients of variation of each assay, followed by a decline in the gonadotropin level. To reduce false positive pulse identification, we additionally required that each pulse consist of at least two consecutive data points, each at least $20 \%$ above baseline, and be at least $2 \mathrm{IU} / \mathrm{L}$ in amplitude $(11,12)$.

To minimize false negative pulse data, we examined the effect of lowering the threshold amplitude required for pulse identification from 2 to $1 \mathrm{IU} / \mathrm{L}$. This was of particular concern because overly restrictive pulse criteria might mask low amplitude pulses that may characterize daytime pubertal or nighttime follicular phase GnRH secretion, thus giving the appearance of diurnal variation where there was none.

Daytime and nighttime mean gonadotropin levels were calculated for each girl with CPP and for each woman in the EFP. To facilitate comparisons between the two groups, only 4-h windows $(2200-0200 \mathrm{~h}$ and $1000-1400 \mathrm{~h})$ were analyzed, despite the availability of extended monitoring in the adult women. Determination of $\mathrm{LH}$ pulse amplitude required the presence of both an identifiable nadir and peak.

LH pulse frequency was expressed as pulses per patient-h. However, the boundaries of the sampling windows in the children with CPP create special problems for pulse identification. In some cases, pulses were truncated by the beginning or end of the sampling period, thus preventing them from meeting pulse criteria. The effect of this truncational artifact was to lower the estimate of pulse frequency during the first and last hour of each sampling window and thus during the 4-h window as a whole. To overcome this difficulty, a corrected pulse frequency was derived from the middle $2 \mathrm{~h}$ of each sampling period, inasmuch as these were largely free of such truncational artifacts and thus provided the most accurate estimate of GnRH pulse frequency. Pulses first were identified from the crude 4-h data using the methods described. The peak of a pulse then was defined unambiguously as the highest concentration attained in a pulse or, where there were two peak data points of equal concentration, the first of these. Any peak thus identified between 2300 to 0050 $\mathrm{h}$ and 1100 to $1250 \mathrm{~h}$ inclusive was then assigned uniquely to the $2-\mathrm{h}$ window and the pulse frequency expressed as pulses per patient-h. Because data points antecedent and subsequent to the 4-h sampling windows were available for analysis in the eight EFP women who underwent continuous sampling, no such correction was required of their data. FSH pulse frequencies and amplitudes were not analyzed for either group because of the large number of patients without identifiable FSH pulses.

Correlation of neuroendocrine secretion with advancing sexual maturation. To track the development of neuroendocrine function across puberty in the girls with CPP, we explored associations between clinical indices of pubertal development and changes in gonadotropin secretion. These clinical indices included breast development, skeletal maturation, and the presence or absence of a history of menses.

Statistics. Paired two-tailed $t$ testing was used to analyze diurnal differences in mean gonadotropin levels, LH pulse amplitude, and LH pulse frequency. The Wilcoxen rank sum test was used to compare the group of 39 girls with CPP with the eight normal women in the EFP as well as for comparisons between subgroups of girls with CPP.

\section{RESULTS}

Clinical and Biochemical Indices. Girls with CPP. The mean chronologic age of the 39 CPP girls was $6.4 \pm 0.3$ y with a range of 1.6 to $9.2 \mathrm{y}$. All had exhibited clinical evidence of sexual development (i.e. breast development at Tanner stage 2 or greater) before 8 y of age and all had a normal cranial CT scan. The mean bone age of the group was $10.0 \pm 0.4$ y (range 2.6$13.2 \mathrm{y}$ ). On average, the girls were $2.1 \mathrm{SD}$ above the mean height for chronologic age. All girls had either stage 3 or 4 breast development. Vaginal bleeding had occurred in six girls.

Although estradiol levels were $<70 \mathrm{pmol} / \mathrm{L}$ in 30 of 38 girls (ranging from $90-210 \mathrm{pmol} / \mathrm{L}$ in the remaining eight), the mean vaginal maturation index score was $50.7 \pm 2.6$, indicative of an estrogen effect on the vaginal mucosa. Progesterone levels were $<2.3 \mathrm{nmol} / \mathrm{L}$ in all girls.

Women in EFP. The mean chronologic age of the EFP women was $27.1 \pm 3.5 \mathrm{y}$ with a range of 18 to $35 \mathrm{y}$. Normal ovulatory function was confirmed in prior and study cycles by daily sampling of LH, FSH, estradiol, and progesterone, as previously reported (3). During the EFP studies, at least one and as many as four of the six estradiol levels obtained were below the level of assay detectability in seven of the eight women. In all, 13 of 36 samples were below the limits of assay detectability, whereas the remaining 23 ranged from 120 to $270 \mathrm{pmol} / \mathrm{L}$. No diurnal pattern could be recognized in the estradiol levels. Progesterone levels were $<2 \mathrm{nmol} / \mathrm{L}$ in all eight women.

Gonadotropin Data. Girls with CPP. LH secretion showed 


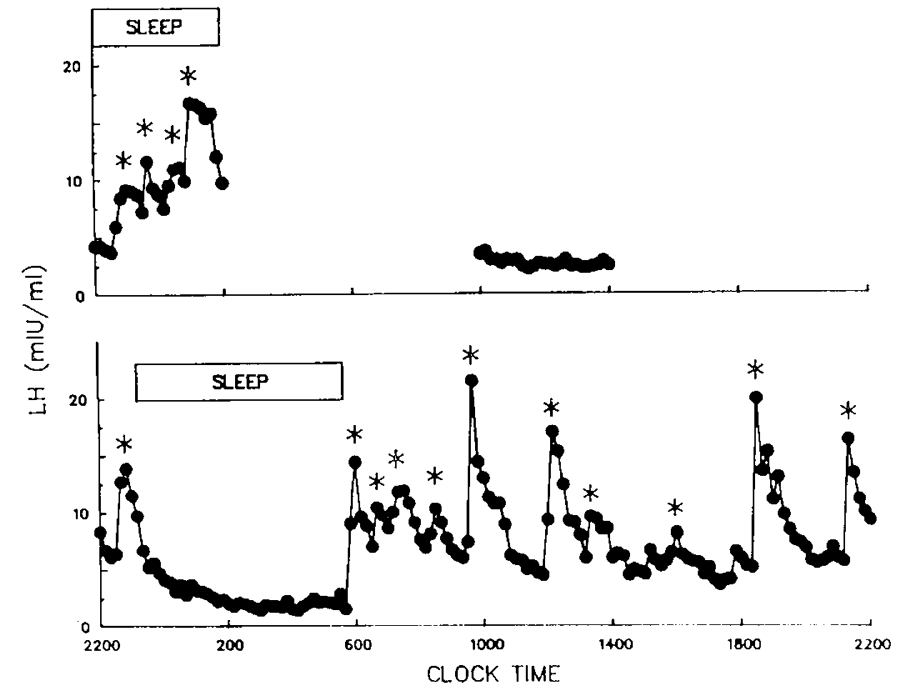

Fig. 1. LH puise patterns in a girl with CPP (top) and a normal woman in the EFP of the menstrual cycle (bottom). Asterisks denote objectively determined LH pulses. The girl with CPP demonstrates increased mean LH levels and LH pulse amplitude and frequency during nighttime monitoring. In contrast, the woman in the EFP exhibits diminished LH secretory activity at night with an increase in mean levels and LH pulse frequency during the day.
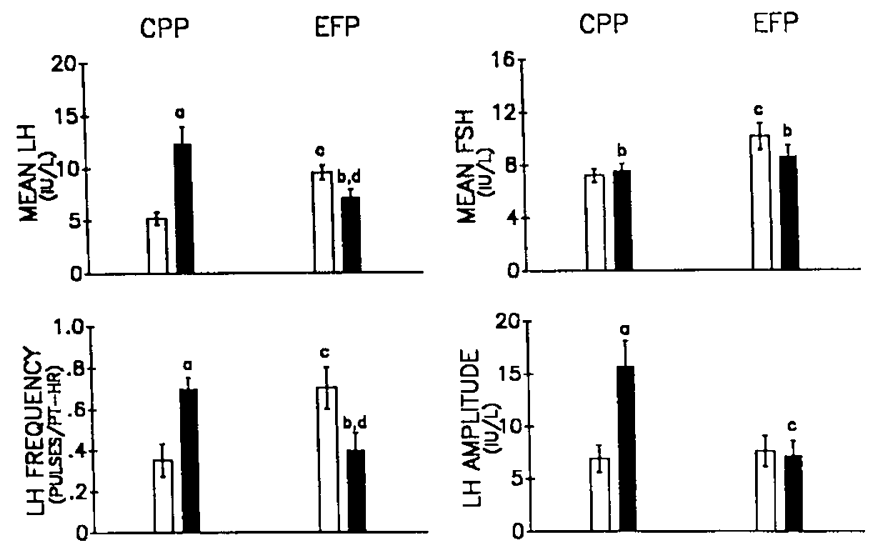

Fig. 2. Daytime (open bars) and nighttime (solid bars) gonadotropin pulse indices in 39 girls with precocious puberty (CPP) and eight women in the EFP of the menstrual cycle. Mean gonadotropin levels and LH pulse amplitude are expressed in IU/L and are derived from 4-h sampling windows (1000-1400 h, 2200-0200 h) for each group. LH pulse frequency is expressed in pulses/patient-h and has been corrected for truncational artifact (see Methods) in the patients with CPP. Data expressed as mean \pm SEM. $a, p \leq 0.01$ when compared with daytime value; $b, p \leq 0.05$ when compared with daytime value; $c, p \leq 0.01$ when compared with CPP group; $d, p \leq 0.05$ when compared with CPP group.

striking sleep-associated differences in the girls with CPP (Fig. 1). Mean nighttime LH levels were more than twice as high as those during the day due to nocturnal increases in both $\mathrm{LH}$ pulse amplitude and frequency (Fig. 2).

All girls exhibited at least one LH pulse and as many as four LH pulses during the 4-h nocturnal monitoring period, whereas 17 girls had no daytime pulsatile secretion. The remaining 22 patients exhibited from one to five daytime pulses. Thus, there were a total of 89 nocturnal and 39 daytime pulses for the group as a whole for a mean nocturnal pulse frequency of $0.58 \pm 0.04$ versus $0.25 \pm 0.05$ pulse/patient-h during the day $(p \leq 0.01)$ These corresponded to interpulse intervals of $105 \mathrm{~min}$ at night versus 240 min during the day. When only the middle $2 \mathrm{~h}$ of each sampling period were considered, i.e. time periods that were largely free of such truncational artifacts (see Methods) and thus provided a more accurate estimate of $\mathrm{GnRH}$ pulse frequency, mean LH pulse frequencies rose to $0.70 \pm 0.05$ pulse/patient-h at night versus $0.35 \pm 0.08$ pulse/patient-h during the day $(p \leq$ $0.01)$. The derived mean nocturnal and daytime interpulse intervals fell to 87 and $167 \mathrm{~min}$, respectively (night versus day, $p \leq$ 0.01 ).

Despite these diurnal differences in LH pulsatility, only 26 of 39 girls demonstrated pulsatile secretion of FSH at night and only 13 of 39 during the day. Mean nocturnal FSH levels were only slightly higher than daytime levels (Fig. 2). Although these diurnal changes were small, they were quite consistent and thus achieved statistical significance $(p \leq 0.05)$. However, the more striking differences in LH levels resulted in a nearly 2-fold higher $\mathrm{LH}$ :FSH ratio during the night than during the day $(1.7 \pm 0.2$ versus $0.9 \pm 0.2, p \leq 0.01$ )

Women in EFP. The women in the EFP of the menstrual cycle also demonstrated sleep-associated variation in LH secretion, but the pattern was opposite that of the girls with CPP, as illustrated by the two patients in Figure 1. In contrast to the girls with CPP, mean LH levels in the women in the EFP were higher during the day than during the night. This appeared to be due to sleepassociated slowing of $\mathrm{LH}$ pulse frequency in the women in the EFP, which resulted in a significantly higher $\mathrm{LH}$ pulse frequency during the day. There was no significant day-night difference in LH pulse amplitude (Fig. 2). The corresponding daytime and nighttime interpulse intervals were 87 and $150 \mathrm{~min}$.

As with the girls with CPP, women in the EFP demonstrated less diurnal variation in FSH levels than in LH levels (Fig. 2). However, in contrast to the CPP girls, FSH levels in the EFP women tended to be higher during the day (Fig. 2). As a result, the LH:FSH ratio again showed diurnal differences and, in contrast to the CPP girls, was significantly higher during the day $(1.1 \pm 0.2$ versus $0.8 \pm 0.2, p \leq 0.05)$. Pulsatile FSH secretion was evident in only three of eight women at night and in six of eight women during the day.

Correlation of neuroendocrine secretion with advancing sexual maturation. There was a consistent but not statistically significant trend toward higher mean daytime $\mathrm{LH}$ levels and daytime $\mathrm{LH}$ pulse frequency with progressive maturation as measured by breast development or bone age advancement. In the girls with a history of menses, there was a loss of diurnal variation in mean LH levels and in LH pulse frequency. None of the subgroups demonstrated diurnal variation in FSH secretion, and mean levels tended to be higher in the more advanced girls.

Impact of varying pulse identification criteria. Lowering the threshold amplitude requirement from 2 to $1 \mathrm{IU} / \mathrm{L}$ resulted in the identification of an additional 10 nighttime and 27 daytime pulses in the CPP girls and only one additional daytime pulse in the EFP women. There were still significantly more nocturnal than daytime pulses in the CPP girls (99 versus $66, p \leq 0.01$ ) and more daytime than nighttime pulses in the EFP women (23 versus $13, p \leq 0.01$ ).

\section{DISCUSSION}

In this study, we have attempted to characterize the maturation of the hypothalamic-pituitary axis by contrasting the neurosecretory dynamics of $\mathrm{GnRH}$ of two groups-pubertal girls and adult women-with similar sex steroid milieus. The use of peripheral concentrations of $\mathrm{LH}$ as an index of hypothalamic GnRH secretory activity is supported by previous studies in $\mathrm{GnRH}$-deficient patients and animal models using exogenously administered $\mathrm{GnRH}$, as well as in intact animal models with direct measurements of hypophyseal portal levels of GnRH (13-16). Our data suggest that there is striking diurnal variation in GnRH secretion during early sexual maturation, as reflected by a 2-fold nighttime rise in mean LH levels, pulse amplitude, and pulse frequency. This pattern is in sharp contrast to that seen in women in the EFP of the menstrual cycle who, under qualitatively similar conditions of ovarian steroid production, display sleep-related 
slowing of GnRH secretion. Secretion of FSH, on the other hand, lacks the striking pulsatility and diurnal changes in mean levels characteristic of $\mathrm{LH}$. The combination of these changes thus results in alternating periods of relative LH and FSH predominance during both sexual development and the folliculogenic portion of the menstrual cycle.

These findings regarding changes in the pattern of diurnal variation in gonadotropin secretion across development and, more specifically, in GnRH-induced LH pulse frequency contrast with those of other investigators who also have attempted to control for the influence of sex steroids on the maturation of the hypothalamic-pituitary axis by using the sex steroidless clinical model of girls with gonadal dysgenesis (17-19). In contrast to our study groups, these study populations demonstrate rises in mean gonadotropin levels and pulse amplitude, but no changes in overall gonadotropin pulse frequency, with increasing age. Furthermore, no diurnal differences in gonadotropin pulse frequency are seen in the hypogonadal patients, although findings regarding diurnal variation in gonadotropin pulse amplitude are inconsistent. Thus, these other investigators have concluded that developmental changes in gonadotropin secretory dynamics are solely mediated by changes in gonadotropin and, by extension, GnRH pulse amplitudes.

A combination of methodologic and model-related differences appear to underlie the contrasting observations regarding maturational and sleep-associated changes in neuroendocrine function in our study and those in patients with gonadal dysgenesis. One study of patients with gonadal dysgenesis used the sum of $\mathrm{LH}$ and FSH pulses to define pulse frequency in their study population (17). In fact, $\mathrm{LH}$ pulse frequency increased with increasing age in these patients, whereas FSH pulse frequency decreased, suggesting that maturation of neuroendocrine function is indeed characterized by changes in GnRH pulse frequency. With respect to the contrasting findings between these studies and our own regarding sleep-associated changes in neurosecretory activity with progressive maturation, it might be argued that the inability to identify low-amplitude LH pulses in our study (a problem potentially obviated by the use of hypogonadal patients with exaggerated pulses) gave the impression of diurnal variation in neurosecretory activity where in actuality there was none. This seems unlikely for three reasons. First, our women in the EFP, who showed no diurnal difference in LH pulse amplitude, nevertheless exhibited diurnal differences in mean $\mathrm{LH}$ levels and $\mathrm{LH}$ pulse frequency. Second, in the girls with CPP, the application of less stringent pulse criteria failed to eradicate diurnal differences in LH pulse frequency. And third, recent studies elsewhere with a highly sensitive immunoradiometric LH assay failed to demonstrate daytime pulsatile secretion of $\mathrm{LH}$ in early pubertal boys (20). Finally, although the presence of ovarian steroid hormones in our patients and their absence in the patients with gonadal dysgenesis may explain absolute differences in gonadotropin pulse amplitude and frequency between the two models, this does not account for the observed maturational and diurnal differences in gonadotropin secretion exhibited by our two study populations, in whom sex steroid levels were comparable. Other model-related factors, such as the effects of nonsteroidal ovarian hormones on neuroendocrine activity or a fundamental alteration in CNS function due to the underlying genetic defect inherent in patients with gonadal dysgenesis, thus may be responsible for the conflicting findings in our study and those in patients with gonadal dysgenesis.

The basis for the apparent differential impact of sleep on GnRH-induced LH secretion at different stages of development in patients with an intact hypothalamic-pituitary-gonadal axis remains to be elucidated. Other investigators have demonstrated that peak GnRH pulse frequencies during puberty are achieved somewhat later at night than our nighttime sampling window covered (21). Thus, rates of hypothalamic discharge at night during puberty may approach the "free-running" or castrate GnRH pulse frequency, suggesting a relative refractoriness of
GnRH secretion to sex-steroid feedback at this stage. Such a hypothesis is supported by the facts that: 1) acute infusions of pubertal levels of testosterone fail to eradicate the nocturnal rise in LH in early puberty $(21,22)$ and 2$)$ naloxone blockade of the endogenous opiate system, through which the negative feedback effects of sex steroids are thought to be mediated, fails to induce a further rise in GnRH pulse frequency during the day or night until mid- to late puberty $(23,24)$. These data suggest that sex steroids exert little influence on GnRH secretion in early puberty. In contrast, although women in the EFP of the menstrual cycle also are resistant to further stimulation by opiate blockade during the day (25), they do respond to opiate antagonists with nocturnal enhancement of GnRH-induced LH secretion (26), despite an absence of diurnal variation in estrogen levels. Thus, changes in endogenous opiate tone independent of the sex steroid milieu appear to mediate, in part, sleep-associated changes in gonadotropin secretion across development.

The association of sleep with alterations in GnRH secretion has led to a search for sleep-associated substances with an impact on neuroendocrine function. Melatonin, which is an important modulator of neuroendocrine function in many animals (27) and possibly in man (28), has been shown in humans to have a diurnal and developmental pattern of secretion with levels highest at night and a gradual fall in these nocturnal levels with progressive maturation $(29,30)$. Nevertheless, recent studies failed to demonstrate changes in the diurnal pattern of melatonin secretion across the menstrual cycle despite apparent changes in GnRH pulse frequency (31). Prolactin, with a similar nocturnal augmentation in levels, also failed to vary significantly during the menstrual cycle (31). These data thus do not support a role for either melatonin or prolactin in the ontogeny of sleepassociated changes in GnRH secretion. DSIP, a naturally occurring neuropeptide that induces sleep in mammals, recently has been shown to elicit LH but not FSH secretion in rats, apparently through GnRH-dependent pathways $(32,33)$. These studies have involved the administration of pharmacologic doses of DSIP in a nonphysiologic manner, and further work will be required to determine the relevance of DSIP to neuroendocrine maturation in humans.

In contrast to control of GnRH-induced LH secretion, control of FSH secretion across sexual development in the female remains largely undefined. In our patients with CPP, the relatively minor changes in FSH levels across the day failed to parallel the profound alterations in GnRH-induced LH secretion. Similarly, FSH levels in our patients in the EFP showed less diurnal variation than LH levels. Consequently, these changes in gonadotropin levels resulted in alternating periods of relative $\mathrm{LH}$ and FSH predominance during the study periods. Such variation may have important ramifications on gonadal function and development, as evidenced by the observation that many women with polycystic ovarian disease exhibit sustained elevations in the LH:FSH ratio (34). The possible roles of gonadal peptides such as inhibin and activin and/or a separate putative FSHspecific hypothalamic releasing factor in the ontogeny of FSH secretion across sexual development await a fuller characterization.

Certain limitations in our study deserve mention. First, we have studied girls with precocious puberty rather than children undergoing normally timed puberty. Such a substitution seems reasonable in view of the absence of evidence to suggest that the maturational process in girls with CPP is in any way abnormal save for the timing of its onset. In addition, the difficulty in justifying such extensive and invasive investigations in children with no evident abnormalities of puberty traditionally has led to the use of various clinical models (e.g. girls with gonadal dysgenesis, boys with delayed puberty) to study the maturational process in the past. Second, the small number of patients in each study group places important limitations on the interpretation of data, particularly in view of the short sampling windows used in the girls with CPP. Nonetheless, these represent the largest 
groups of pubertal children and normal women to date to be studied so intensively and in so truly a parallel manner. Third, we have assumed that $\mathrm{LH}$ secretion is an accurate reflection of hypothalamic GnRH secretory activity. Although abundant experimental data suggests that this is true $(13,14)$, these data rest on technically difficult manipulations, and conflicting data does exist $(14,35)$. Fourth, since we did not perform EEG studies, we cannot objectively document sleep in all patients during nighttime sampling. Our interchangeable use of the terms "nocturnal," "nighttime," and "sleep-associated," is based on previous studies $(1,2)$ in which a specific relationship between sleep and gonadotropin secretion has been documented. Fifth, although we sought to match our study populations for sex hormone levels, limitations in the sensitivity of our estradiol assay may have masked differential effects of estradiol at low concentrations. Finally, although stratification of the pubertal group resulted in trends in gonadotropin secretion consistent with a process of neuroendocrine maturation during puberty, these changes failed to achieve statistical significance. Difficulties in applying traditional methods of pubertal staging in our pubertal population and an apparent lack of diversity within this population, at least with respect to Tanner staging, contributed to this problem. A truly definitive description of neuroendocrine maturation will require long-term, longitudinal studies in children undergoing a normal puberty.

\section{REFERENCES}

1. Boyar R, Finkelstein J, Roffwarg H, Kapen S, Weitzman E, Hellman L 1972 Synchronization of augmented luteinizing hormone secretion with sleep during puberty. N Engl J Med 287:582-586

2. Kapen S, Boyar R, Perlow M, Hellman L, Weitzman ED 1973 Luteinizing hormone: changes in secretory pattern during sleep in adult women. Life Sci 13:693-701

3. Filicori M, Santoro N, Merriam GR, Crowley WF 1986 Characterization of the physiological pattern of episodic gonadotropin secretion throughout the human menstrual cycle. J Clin Endocrinol Metab 62:1136-1144

4. Goodman RL, Karsch FJ 1980 Pulsatile secretion of luteinizing hormone: differential suppression by ovarian steroids. Endocrinology 107:1286-1290

5. Tanner JM, Marshall WA 1969 Variations in pattern of pubertal changes in girls. Arch Dis Child 44:291-303

6. Meisels A 1967 The maturation value. Acta Cytol 11:249

7. Greulich WW, Pyle SI 1959 Radiographic Atlas of Skeletal Development of the Hand and Wrist. Stanford University Press, Stanford, California

8. Filicori M, Crowley WF 1983 Hypothalamic regulation of gonadotropin secretion in women. In: Norman RL (ed) Neuroendocrine Aspects of Regulation. Academic Press, New York, pp 285-295

9. Filicori M, Butler JP, Crowley WF 1984 Neuroendocrine regulation of the corpus luteum in the human. J Clin Invest 73:1638-1647

10. Crowley WF, Beitins IZ, Vale W, Kliman B, Rivier J, Rivier C, McArthur J 1980 The biologic activity of a potent analogue of gonadotropin-releasing hormone in normal and hypogonadotropic men. N Engl J Med 302:10521057

11. Santen RJ, Bardin CW 1973 Episodic luteinizing hormone secretion in man. Pulse analysis, clinical interpretation, physiological mechanisms. J Clin Invest 52:2617-2628

12. Spratt DI, Carr DB, Merriam GR, Scully RE, Rao PN, Crowley WF 1987 The spectrum of abnormal patterns of gonadotropin-releasing hormone secretion in men with idiopathic hypogonadotropic hypogonadism: clinical and laboratory correlations. J Clin Endocrinol Metab 64:283-291

13. Belchetz PE, Plant TM, Nakai Y, Keogh EJ, Knobil E 1978 Hypophyseal responses to continuous and intermittent delivery of hypothalamic gonadotropin-releasing hormone. Science 202:631-633

14. Levine JE, Pau KF, Ramirez VD, Jackson GL 1982 Simultaneous measurement of luteinizing hormone-releasing hormone and luteinizing hormone release in unanesthetized, ovariectomized sheep. Endocrinology 111:14491455

15. Wilson RC, Kesner JS, Kaufman JM, Uemura T, Akema T, Knobil E 1984 Central electrophysiologic correlates of pulsatile luteinizing hormone secretion in the rhesus monkey. Neuroendocrinology 39:256-260

16. Crowley WF, Filicori M, Spratt DI, Santoro NF 1985 The physiology of gonadotropin-releasing hormone $(\mathrm{GnRH})$ secretion in men and women. Recent Prog Horm Res 41:473-531

17. Ross JL, Loriaux DL, Cutler GB 1983 Developmental changes in neuroendocrine regulation of gonadotropin secretion in gonadal dysgenesis. J Clin Endocrinol Metab 57:288-293

18. Hosada A, Fujieda K, Yuri K, Okuno A, Matsuura N 1989 Developmental changes of pulsatile LH secretion in Turner syndrome. 7 Ist Annual Meeting of the Endocrine Society, Seattle, Washington (abstr)

19. Hosada A, Fujieda K, Matsuura N, Okuno A, Yuri K 1989 Developmental change of gonadotropin $(\mathrm{Gn})$ secretory dynamics in Turner syndrome. Horm Res 31:4(abstr)

20. Wennink JMB, Delemarre-van de Waal HA, van Kessel H, Mulder GH, Foster JP, Schoemaker J 1988 Luteinizing hormone secretion patterns in boys at the onset of puberty measured using a highly sensitive immunoradiometric assay. J Clin Endocrinol Metab 67:924-928

21. Hale PM, Khoury SA, Foster CM, Beitins IZ, Hopwood NJ, Marshall JC, Kelch RP 1988 Increased luteinizing hormone pulse frequency during sleep in early to midpubertal boys: effects of testosterone infusion. J Clin Endocrinol Metab 66:785-791

22. Foster CM, Hassing JM, Mendes TM, Hale PM, Padmanabhan V, Hopwood NJ, Beitins IZ, Marshall JC, Kelch RP 1989 Testosterone infusion reduces nocturnal luteinizing hormone pulse frequency in pubertal boys. $J$ Endocrinol Metab 69:1213-1220

23. Veldhuis JD, Rogol AD, Johnson ML, Dufau ML 1983 Endogenous opiates modulate the pulsatile secretion of biologically active luteinizing hormone in man. J Clin Invest 72:2031-2040

24. Sauder SE Case GD Hopwood NJ, Kelch RP, Marshall JC 1984 The effects of opiate antagonism on gonadotropin secretion in children and in women with hypothalamic amenorrhea. Pediatr Res 18:322-328

25. Quigly ME, Yen SCC 1980 The role of endogenous opiates on LH secretion during the menstrual cycle. J Clin Endocrinol Metab 51:179-181

26. Rossmanith WG, Yen SSC 1987 Sleep-associated decrease in luteinizing hormone pulse frequency during the early follicular phase of the menstrual cycle: evidence for an opioidergic mechanism. J Clin Endocrinol Metab 65:715-718

27. Reiter RJ 1980 The pineal and its hormones in the control of reproduction in mammals. Endocr Rev 1:109-131

28. Brzezinski A, Lynch HJ, Wurtman RJ, Seibel MM 1987 Possible contribution of melatonin to the timing of the luteinizing hormone surge. [letter] $\mathrm{N}$ Eng J Med 316:1550-1551

29. Attanasio A, Borrelli P, Gupta D 1985 Circadian rhythms in serum melatonin from infancy to adolescence. J Clin Endocrinol Metab 61:388-390

30. Waldhauser F, Frisch H, Waldhauser M, Weiszenbacher $G$, Zeitlhuber U Wurtman RJ 1984 Fall in nocturnal serum melatonin during prepuberty and pubescence. Lancet 1:362-365

31. Brzezinski A, Lynch HJ Seibel MM, Deng MH, Nader TM, Wurtman RJ 1988 The circadian rhythm of plasma melatonin during the normal menstrual cycle and in amenorrheic women. J Clin Endocrinol Metab 66:891895

32. Iyer KS, McCann SM 1987 Delta sleep inducing peptide (DSIP) stimulates the release of LH but not FSH via a hypothalamic site of action in the rat. Brain Res Bull 19:535-538

33. Sahu A, Kalra SP 1987 Delta sleep-inducing peptide (DSIP) stimulates LH release in steroid-primed ovariectomized rats. Life Sci 40:1201-1206

34. Yen SSC, Vela P, Rankin J 1970 Inappropriate secretion of follicle-stimulating hormone and luteinizing hormone in polycystic ovarian disease. $\mathbf{J}$ Clin Endocrinol Metab 30:435-442

35. Clarke IJ, Cummins JT 1987 The significance of small pulses of gonadotropinreleasing hormone. J Endocrinol 113:413-418 